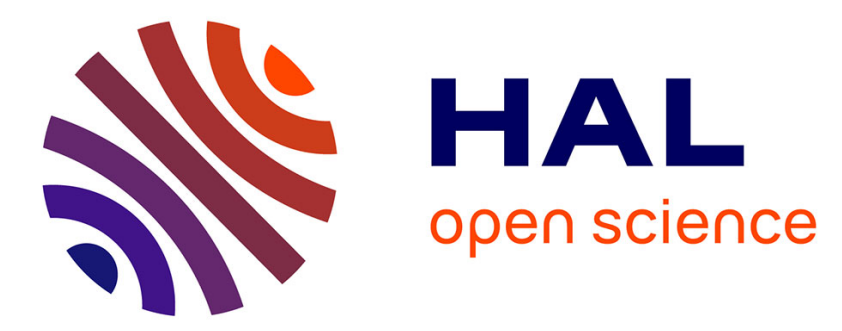

\title{
Tailoring simultaneous photonic and phononic band gaps
}

\author{
S. Sadat Saleh, S. Benchabane, F.I. Baida, M.-P. Bernal, V. Laude
}

\section{To cite this version:}

S. Sadat Saleh, S. Benchabane, F.I. Baida, M.-P. Bernal, V. Laude. Tailoring simultaneous photonic and phononic band gaps. Journal of Applied Physics, 2009, 106, pp.074912. 10.1063/1.3243276 . hal-00447206

\section{HAL Id: hal-00447206 \\ https://hal.science/hal-00447206}

Submitted on 15 May 2021

HAL is a multi-disciplinary open access archive for the deposit and dissemination of scientific research documents, whether they are published or not. The documents may come from teaching and research institutions in France or abroad, or from public or private research centers.
L'archive ouverte pluridisciplinaire HAL, est destinée au dépôt et à la diffusion de documents scientifiques de niveau recherche, publiés ou non, émanant des établissements d'enseignement et de recherche français ou étrangers, des laboratoires publics ou privés. 


\title{
Tailoring simultaneous photonic and phononic band gaps
}

\author{
Said Sadat-Saleh, Sarah Benchabane, ${ }^{\text {a) }}$ Fadi Issam Baida, Maria-Pilar Bernal, \\ and Vincent Laude \\ Institut FEMTO-ST, Université de Franche-Comté, CNRS, ENSMM, UTBM 32 Avenue de l'Observatoire, \\ F-25044 Besançon Cedex, France
}

(Received 16 April 2009; accepted 8 September 2009; published online 15 October 2009)

\begin{abstract}
The simultaneous existence of photonic and phononic band gaps opens up many possibilities for enhancing acousto-optical interactions at a common wavelength scale. We term such structures phoxonic crystals. By computing the existence and dependence of phoxonic band gaps on the choice of lattice and unit cell, we obtain a hierarchy of two-dimensional phoxonic crystal structures. The single-atom hexagonal and square lattices, and some multiple-atom hexagonal lattices, including honeycomb and heterometric lattices, are investigated. For definiteness, arrays of air holes in lithium niobate are considered in the computations. It is observed that decreasing the symmetry of the lattice by adding atoms of different sizes inside the unit cell leads to larger phoxonic band gaps. Examples of designs for operation at an optical wavelength of $1550 \mathrm{~nm}$ are given. The corresponding phononic frequencies are in the gigahertz range. (C) 2009 American Institute of Physics.
\end{abstract}

[doi:10.1063/1.3243276]

\section{INTRODUCTION}

It is well established that band gap effects for the propagation of both light or sound waves can be obtained in artificially engineered periodic materials. In the case of photonic crystals, ${ }^{1,2}$ the propagation medium exhibits a periodic modulation of the refractive index, which can prohibit electromagnetic wave propagation in a specific wavelength range, giving rise to photonic band gaps and allowing to tailor the dispersion properties of light. Phononic crystals, ${ }^{3}$ for their part, are periodic composites made of two or more materials with different elastic constants and densities. They also can prevent elastic wave propagation in given frequency ranges and offer new possibilities of controlling sound propagation. Up to now, the two research fields have been mainly explored in quite an independent fashion. Yet, the possibility of designing and fabricating hypersonic phononic crystals operating in the radio-frequency regime (and therefore presenting lattice parameters smaller than $1 \mu \mathrm{m})^{4}$ has shown that photonic and phononic crystals of comparable dimensions can be made.

A recent trend has emerged, which aims at designing periodic materials capable of controlling simultaneously photon and phonon propagation and jointly confining sound and light. Much similarly to what has occurred with phononic and photonic crystals independently, these "phoxonic" crystals, in addition to an enhancement of the acoustooptical interactions through localization or slow-wave effects, ${ }^{5}$ could be the seat of other unexpected phenomena. Control of photon-phonon interaction at a fundamental level is a most appealing possibility and has already been at the core of some studies in one-dimensional superlattices. ${ }^{6,7}$ Works related to two-dimensional (2D) or three-dimensional

${ }^{a)}$ Electronic mail: sarah.benchabane@femto-st.fr.
(3D) structures, however, remain scarce, except for a growing interest toward light-induced phonons and their management in photonic crystal fibers. ${ }^{8-10}$

Maldovan and Thomas ${ }^{11,12}$ have shown theoretically that phoxonic band gaps can be obtained in 2D square or hexagonal lattice crystals made of air holes in a silicon matrix. Experimental evidence of such a band gap phenomenon has been reported recently in a 3D phoxonic crystal of amorphous silica spheres. ${ }^{13}$ However, these works have focused on simple lattice geometries and there is no guaranty that phoxonic band gaps might be obtained with other materials. There is thus an interest in looking into more complex geometries, e.g., multiple-atom/heterometric structures, is order to tailor phoxonic band gaps.

Some of the most relevant and routinely used materials in conventional optics and high frequency acoustics are quite often anisotropic. Lithium niobate is one of the most striking examples. This material lies at the basis of a significant number of optoelectronic active and passive devices, while remaining a rich playing field for the implementation of proofof-concept optical experiments thanks to its almost unmatched combination of high nonlinear, electro-optical, or acousto-optical coefficients. These intrinsic properties can be allied to the possibilities it offers in terms of waveguiding using well-assessed techniques such as proton exchange or titanium indiffusion. Lithium niobate is also a key material in high frequency acoustics owing to its high piezoelectric coefficients and is frequently used as a substrate for the realization of passive radio-frequency telecommunication filters. Linear photonic and phononic band gap effects were evidenced experimentally in artificial crystals made of air holes in lithium niobate, ${ }^{14,15}$ as well as nonlinear photonic band gaps in a structure made by $2 \mathrm{D}$ periodic poling. ${ }^{16}$ There is however no work reporting on a phoxonic band gap in this particular material. The conditions for the existence of a full photonic band gap in lithium niobate are indeed demanding, 


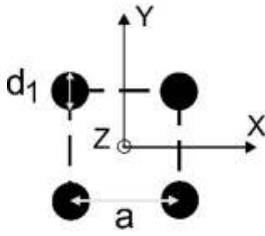

(a)

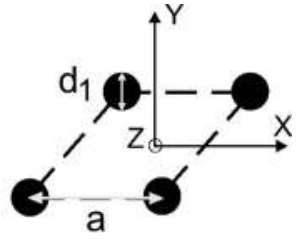

(b)
FIG. 1. 2D periodic array of cylindrical holes in lithium niobate arranged along (a) a square and (b) a hexagonal lattice. The pitch is $a$ and the diameter of the cylinder is $d_{1}$. The crystallographic axes of lithium niobate are shown (the $Z$ axis is aligned with the rod axis). For the TM polarization of light (respectively, TE), the electric field vector (respectively, the magnetic field vector) is aligned with the rod axis.

due to the relatively low refractive index in the near infrared, 2.3 as compared with 3.6 for silicon, for example.

In this paper, we investigate theoretically the existence of phoxonic band gaps in a 2D structure made of air holes in lithium niobate. The most commonly used single-atom lattices, i.e., square and hexagonal, as well as multiple-atom hexagonal lattices, in particular honeycomb and heterometric hexagonal lattices, are considered. In each case, photonic and phononic band structures are computed independently. The entire study remains restricted to cylindrically shaped inclusions. It is observed that true complete phoxonic band gaps, i.e., for all polarizations, are difficult to obtain due to the limited contrast in refractive index. However, complete band gaps for either the transverse electric (TE) or the transverse magnetic (TM) optical polarization can generally be found. As a general rule, it is observed that decreasing the symmetry of the lattice by adding atoms of different sizes inside the unit cell leads to larger phoxonic band gaps. Examples of designs for operation at an optical wavelength of $1550 \mathrm{~nm}$ are given and practically feasible structures are highlighted.

\section{METHODS}

In what follows, we consider 2D photonic and phononic crystal structures composed of air holes in lithium niobate. Holes are considered instead of pillars, since elastic waves could obviously not propagate in vacuum between solid pillars. Moreover, when air is considered as the propagation medium instead of a vacuum, arrays of silicon pillars were found to be inefficient for obtaining phoxonic band gaps. ${ }^{11}$ As compared with other shapes of the scatterers, circular cylindrical holes were found to lead to larger band gaps in all the cases we considered. We then report only results obtained with circular air holes.

Lithium niobate, $\mathrm{LiNbO}_{3}$, is a piezoelectric and anisotropic solid material considered here in its single crystal form ( $3 m$ crystalline symmetry). Holes are supposed to be aligned along the $Z$ crystallographic axis, as depicted in Fig. 1 . Propagation then occurs in the $(X, Y)$ plane. Material constants (optical, elastic, dielectric, and piezoelectric) are taken from Refs. 17 and 18.

The occurrence of phoxonic band gaps is dictated by the simultaneous existence of a complete band gap for phonons and photons. More precisely, we determine and compare photonic and phononic gap maps giving the existence of complete band gaps as a function of the geometry of the periodic structure (usually measured by the hole diameter to pitch ratio).

A number of numerical models are available in the literature to compute photonic and phononic band structures. The computation of band structures in piezoelectric phononic crystals can for instance be achieved by the plane wave expansion (PWE) method ${ }^{19-21}$ or the finite element method with periodic boundary conditions. ${ }^{15,22}$ Phononic band structures in this paper were computed using a homemade PWE code. In the case of photonic crystals, we have used both the PWE method ${ }^{23,24}$ as implemented in the BANDSOLVE code ${ }^{25}$ and a homemade finite difference time domain (FDTD) code. ${ }^{26}$ The PWE photonic code was limited to single-atom and honeycomb lattices while the FDTD photonic code could handle all the lattices considered in this paper. We initially checked that both codes gave consistent results.

\section{SINGLE-ATOM LATTICES}

Figure 1 depicts the square and the hexagonal lattices for 2D arrays of holes in a solid matrix. Figure 2 shows the corresponding photonic and phononic band gaps as a function of $d_{1} / a$, being $d_{1}$ the hole diameter and $a$ the period. In the case of photonic crystals, band gaps are represented as a
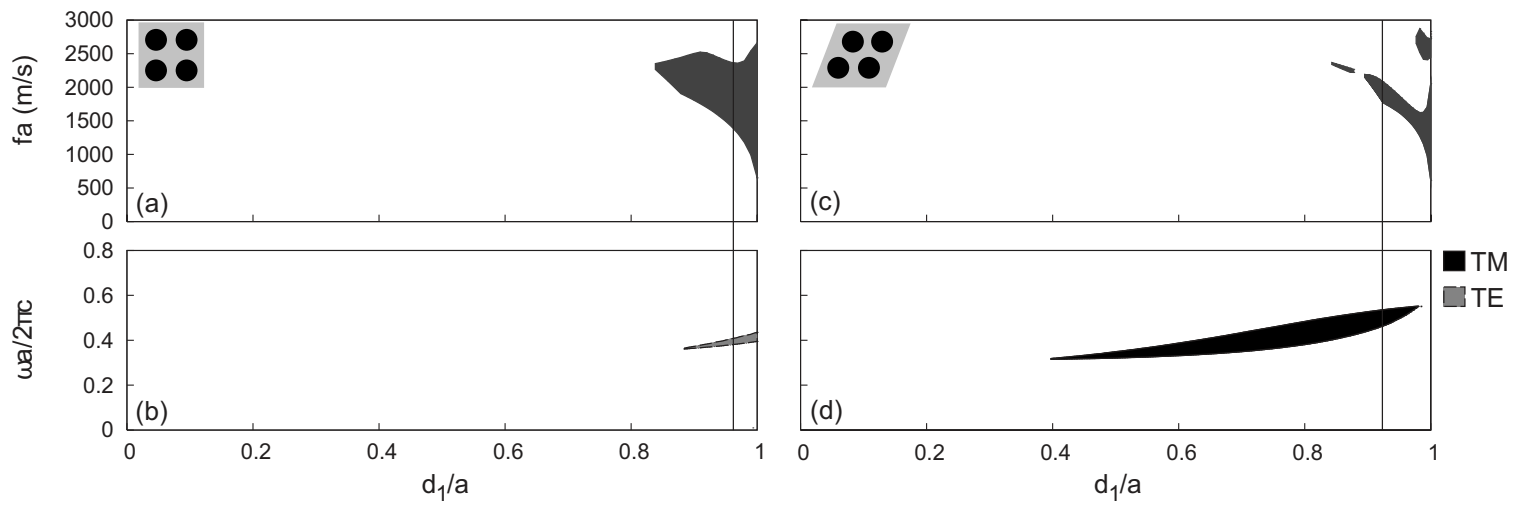

FIG. 2. Phononic (a) and photonic (b) gap maps for a square lattice array of circular air holes in lithium niobate as a function of the ratio $d_{1} / a$. Phononic (c) and photonic (d) gap maps for a hexagonal lattice array of circular air holes in lithium niobate. Photonic gap maps for the TM polarization (black) and TE polarization (light gray) are displayed in units of the normalized frequency $\omega a / 2 \pi c$, with $c$ the speed of light in a vacuum. Phononic gap maps are displayed in units of the product $f a$, with $f$ the acoustic frequency. 
function of the normalized frequency $\omega a / 2 \pi c$, with $\omega$ the optical angular frequency and $c$ the speed of light in a vacuum. For phononic crystals, band gaps are expressed as a function of $f a$, the product of the acoustic frequency $f$ with the pitch of the crystal. We observe that for both the square and the hexagonal lattice there is no complete photonic band gap simultaneously valid for the TE and the TM polarizations. This is not necessarily a drawback since phoxonic crystals can be fabricated on a lithium niobate waveguide produced by annealed proton exchange. ${ }^{14}$ This fabrication method modifies only the ordinary refractive index of the lithium niobate substrate and, as a consequence, there is only one guided light polarization (TE for an $X$-cut substrate and TM for a $Z$-cut substrate). Therefore, as far as the $2 \mathrm{D}$ photonic crystal is concerned, we only need to look for photonic band gaps corresponding to one polarization.

In the square lattice case, a phoxonic band gap is found only for the TE polarization. Figures 2(a) and 2(b) show that both the phononic and photonic gap maps width decrease as $d_{1} / a$ decreases, disappearing for $d_{1} / a=0.884$ in the photonic case and $d_{1} / a=0.837$ in the phononic case, which corresponds to a filling fraction of $61 \%$ and $55 \%$ respectively. Since there is a superposition of both band gaps, the square lattice can be chosen for phoxonic crystal fabrication. In particular, for $d_{1} / a=0.962$, the photonic and phononic gap widths are calculated to be $6.9 \%$ and $53 \%$, respectively. At this operating point, the photonic band gap is found for normalized electromagnetic frequencies between 0.381 and 0.408 and the phononic band gap from $f a=1381 \mathrm{~m} / \mathrm{s}$ to $2353 \mathrm{~m} / \mathrm{s}$. Choosing the telecommunication wavelength of $1550 \mathrm{~nm}$, the lattice period is then fixed to $612 \mathrm{~nm}$ and the hole diameter to $589 \mathrm{~nm}$ resulting in a phononic band gap centered at $3.1 \mathrm{GHz}$. Unfortunately, this phoxonic crystal geometry could be technologically difficult to fabricate due to the fact that holes are very close to each other (the separation is only $23 \mathrm{~nm}$ ).

In the hexagonal lattice case, a phoxonic band gap is found only for the TM polarization. Figures 2(c) and 2(d) show that the phoxonic band gap exists for ratios 0.84 $\leq d_{1} / a \leq 0.97$. In particular, for $d_{1} / a=0.92$, the photonic and phononic gap widths are calculated to be both around $15 \%$. For the telecommunication wavelength of $1550 \mathrm{~nm}$, the lattice period is $770 \mathrm{~nm}$, the hole diameter is $709 \mathrm{~nm}$ and the acoustic band gap is centered at $2.5 \mathrm{GHz}$. The separation between holes is then $61 \mathrm{~nm}$, which relaxes fabrication tolerance as compared with the square lattice example considered above.

Comparing with the results of Maldovan and Thomas for silicon, ${ }^{11,12}$ it can be observed that photonic band gaps are smaller for lithium niobate. Due to the different elastic velocities in these materials, the phononic band gaps are shifted in frequency, but the phononic gap maps are rather similar in relative extent. This significant difference between photonic and phononic crystal properties can be understood as follows. As is well known, the width of photonic band gaps is conditioned by the contrast in the refractive index between the holes $(n=1)$ and the substrate. A material such as lithium niobate is in this respect obviously less advantageous than silicon. The case of phononic crystals is different, since by neglecting the presence of air it can be assumed that there are no elastic waves inside the holes. The scattering of phonons is then provided by the traction-free boundary conditions at the holes, not by a contrast in the material constants of the substrate and the inclusions. Such boundary conditions are independent of the choice of the substrate. Hence, although differences between lithium niobate and silicon will be introduced by their different crystalline symmetry and different distribution of the elastic tensor coefficients, the lattice choice and the unit cell geometry are definitely the dominant parameters.

From a technological point of view, geometries with well separated holes are desirable to increase the fabrication tolerances. Furthermore, the period of the phoxonic crystal is directly governed by the optical wavelength, which in turn indirectly determines the acoustic frequency. Low acoustic frequencies should be privileged for the realization of the phonon sources, for instance based on interdigital transducers, ${ }^{15}$ to be used in conjunction with phoxonic crystals. As we have seen, the square and the hexagonal lattices do not completely meet this objective and we need to look for alternative geometries that provide both high values of the normalized electromagnetic frequencies (e.g., around 0.6) and low phononic frequency band gaps.

\section{MULTIPLE-ATOM HEXAGONAL LATTICES}

From the results of Sec. III, it can be inferred that simple, single-atom lattices are not ideal for the opening of phoxonic band gap in a highly acoustically anisotropic, low refractive index material such as lithium niobate. The idea is then to move to more complex geometries. It has indeed been demonstrated that both photonic and phononic band gaps can be enlarged by breaking or reducing the lattice symmetry. ${ }^{27-30}$ Symmetry breaking can be achieved either by introducing or removing an inclusion in the initial unit cell, or by varying the size of one or some of the inclusions, as in the case of the so-called boron nitride structure. ${ }^{29,31}$ The same principle can hence be applied to ensure the occurrence or the increase in width of phoxonic band gaps. In this section, we investigate lattice geometries based on the honeycomb structure that can itself be seen as a hexagonal lattice with a missing central inclusion and on other modified hexagonal geometries.

Figure 3 displays the gap map obtained in the case of a regular honeycomb structure. A phononic band gap opens for diameter to pitch ratios larger than 0.8 and its width increases with the filling fraction to reach a fractional bandwidth as large as $70 \%$ for $d_{1} / a=0.96$. The photonic band gaps, on the other hand, present much lower fractional bandwidths, whatever the polarization, with a maximum of $10 \%$ for the second TE band. Here again, there is no full photonic band gap, as the gap maps for the TE and TM polarization do not overlap for any value of $d_{1} / a$. Phoxonic band gaps for TE polarized waves can anyway be observed and some of them are completely exploitable from a practical point of view. First, at a fixed wavelength, the higher the photonic band gap central frequency is, the larger the pitch of the structure can be, which allows to relieve quite significantly 


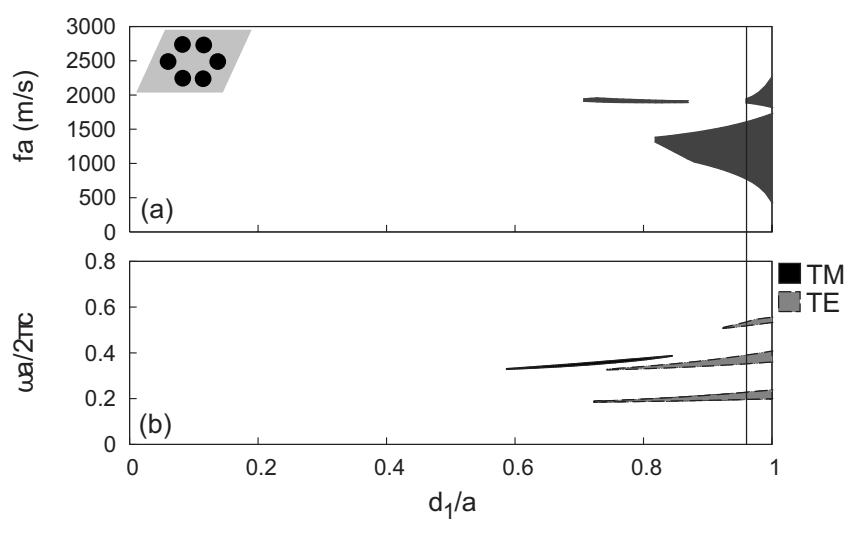

FIG. 3. Phononic (a) and photonic (b) gap maps for a honeycomb lattice array of circular air holes in lithium niobate as a function of the diameter to pitch ratio $d_{1} / a$. Photonic gap maps are given for both the TM (black) and the TE (light gray) polarizations and are displayed in units of the normalized frequency $(\omega a / 2 \pi c)$. Phononic band gaps are displayed in units of the product $f a$, where $f$ is the acoustic frequency.

the constraints related to the crystal fabrication. As an example, the band centered at $\omega a / 2 \pi c=0.371$ is obtained for a crystal exhibiting a pitch of $575 \mathrm{~nm}$ at a $1550 \mathrm{~nm}$ wavelength. Second, the phononic band gap remains at a quite low normalized frequency value. For the $575 \mathrm{~nm}$ pitch previously worked out, the operating frequency of the phononic crystal would be around $2.1 \mathrm{GHz}$, which is very reasonable given the available technologies for acoustic transducer fabrication. However, despite these interesting features, the very low fractional bandwidth of the phoxonic band gap can be a pretty limiting factor, as it will probably make it difficult to observe experimentally. We must then focus on more complicated geometries based on the honeycomb lattice that can provide larger photonic band gaps. The idea is then to take this honeycomb lattice as a starting point and to apply the previously mentioned methods of symmetry breaking to enlarge the phoxonic band gap.

Figure 4(a) illustrates the method we have adopted to this aim. Starting from a honeycomb lattice symmetry, one of the two atoms constituting the unit cell was kept with a fixed diameter at all time, while the diameter of the second atom was varied. The filling factor of the obtained boron nitride structure was therefore not kept constant, conversely to what

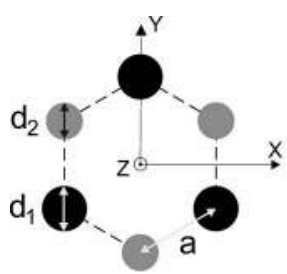

(a)

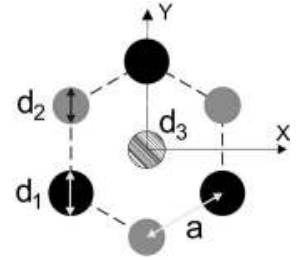

(b)

FIG. 4. 2D periodic array of air holes in lithium niobate arranged according to (a) a boron nitride and (b) a heterometric hexagonal lattice. In (a), the diameter of the black holes, $d_{1}$, is held constant while the diameter of the light gray holes $d_{2}$ is modified to optimize band gap properties. The geometry illustrated in (b) is obtained by adding an additional air hole of diameter $d_{3}$ (hatched gray) at the center of the optimum boron nitride geometry defined by alternating holes of diameters $d_{1}$ (black) and $d_{2}$ (light gray), respectively.

was applied in, ${ }^{28,29}$ for instance, but the pitch was kept the same. We call $\beta$ the ratio between the fixed hole diameter $d_{1}$ and the pitch of the crystal $a$ which is defined as the distance between two neighboring inclusions $\left(\beta=d_{1} / a\right)$. Band diagrams were first computed and the optimum structure determined before applying further modification to the resulting boron nitride lattice by adding another hole with a variable hole at the center of the hexagonal cell. In what follows, this last geometry, depicted in Fig. 4(b), will be referred to as "heterometric hexagonal lattice."

Gap maps for boron nitridelike structures are reported in Fig. 5. Two values for the parameter $\beta$ were chosen, 1 and 1.2 , and the gap maps are plotted as a function of $d_{2} / a$, where $d_{2}$ is the diameter of the variable hole and ranges from 0 to $a-d_{1}$. As a general rule, the symmetry breaking induced by varying the diameter of one hole out of two results in the opening of photonic band gaps for the TM polarization as can be seen in Figs. 5(b) and 5(d) (for $0.55 \leq d_{2} / a \leq 0.90$ when $\beta=1$ and for $0.47 \leq d_{2} / a \leq 0.80$ when $\beta=1.2$ ). For $\beta$ $=1$, no significant impact can be observed for the phononic band diagram. The advantage of the boron nitride structure over a standard honeycomb geometry from an elastic wave point of view becomes more evident when parameter $\beta$ is varied. When $\beta=1.2$ [Fig. 5(d)], wide phononic band gaps centered around $f a=1129 \mathrm{~m} / \mathrm{s}$ open for $d_{2} / a$ larger than 0.5 . The range of existence of photonic band gaps for TE polar-
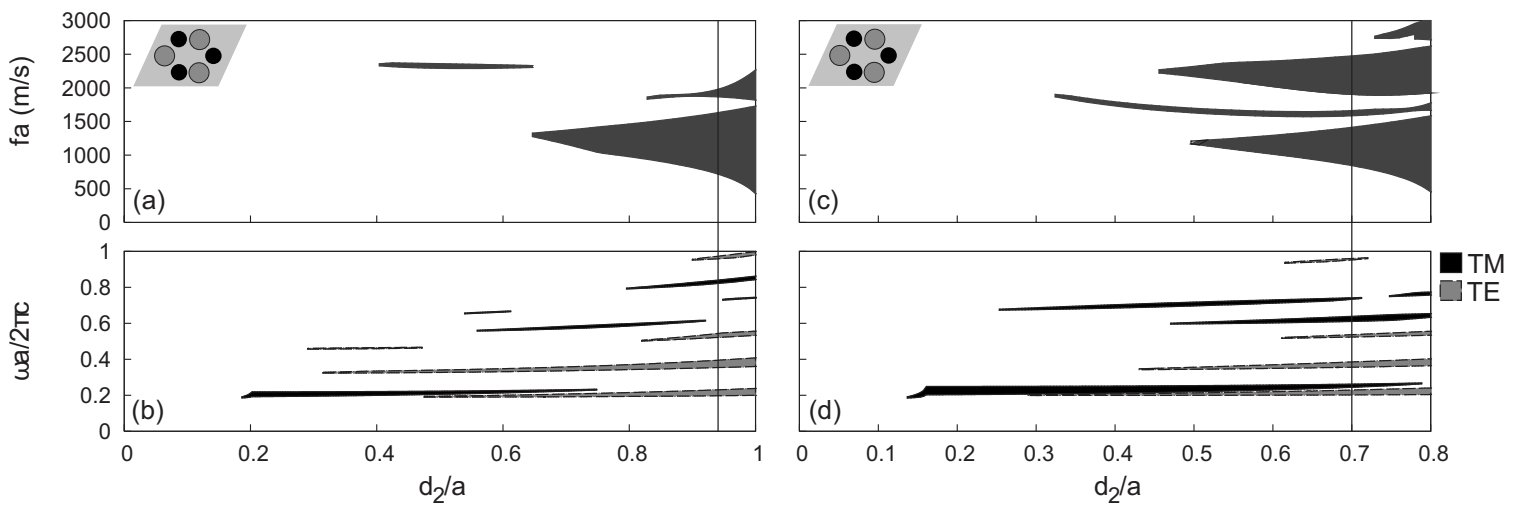

FIG. 5. Phononic (a) and photonic (b) gap maps for boron nitridelike crystals made of circular air holes in lithium niobate, as a function of the diameter to pitch ratio $d_{2} / a$, for $\beta=d_{1} / a=1$. [(c) and (d)] Same but for $\beta=1.2$. Photonic gap maps are given for both the TM (black) and the TE (light gray) polarizations and are represented in units of the normalized frequency $(\omega a / 2 \pi c)$. Phononic band gaps are represented in units of the $f a$ product, where $f$ is the acoustic frequency. 


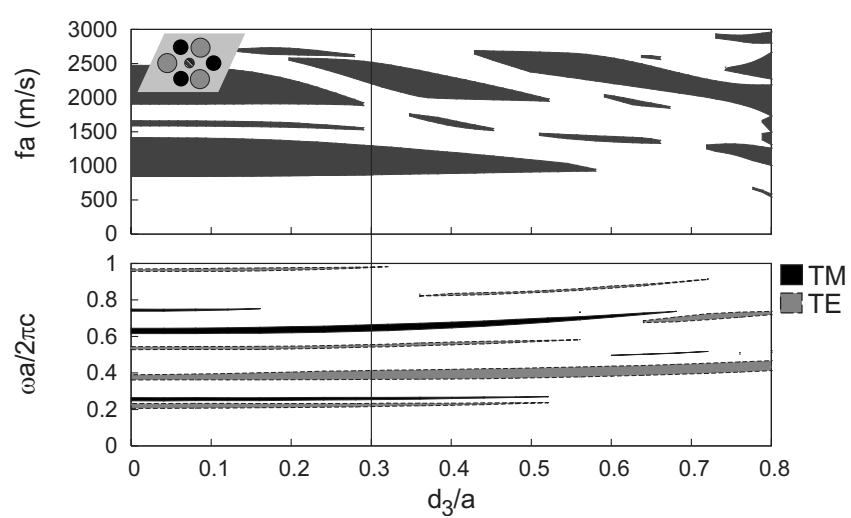

FIG. 6. Phononic (a) and photonic (b) gap maps for heterometric hexagonal lattice crystals made of circular air holes in lithium niobate as a function of the diameter to pitch ratio $d_{3} / a$. Photonic gap maps are given for both the TM (black) and the TE (light gray) polarizations and are represented in units of the normalized frequency $\omega a / 2 \pi c$. Phononic gap maps are represented in units of the product $f a$, where $f$ is the acoustic frequency.

ized waves is however drastically reduced, by almost a factor of 2 , as they range from $d_{2} / a=0.44$ to $d_{2} / a=0.8$, versus 0.33 to 1 for $\beta=1$. A slight increase in the TM gap width can also be observed $(2.3 \%$ versus $4.5 \%)$. These structures hence allow for the opening of several phoxonic band gaps for both $\mathrm{TE}$ and TM polarized waves, though independently. If some of these bands do comply with the technological constraints previously mentioned for the usual honeycomb lattice crystal, the consideration of rather large $a / \lambda$ is found to conflict with the enlargement of photonic band gaps. For instance, if $a / \lambda \approx 0.6$ is chosen, the maximum photonic fractional bandwidth remains quite small, of the order of $4 \%$ in the best case, i.e., for $\beta=1.2$ and $d_{2} / a=0.7$.

The boron nitridelike geometry was therefore further modified through the addition of an inclusion with varying diameter $d_{3}$ at the center of the hexagonal unit cell to form a so-called heterometric hexagonal lattice, as illustrated in Fig. 4(b). The diameters $d_{1}$ and $d_{2}$ of the holes constituting the boron nitride structure were fixed at the optimum values of 1.2 and 0.7 deduced from the previous set of simulations and gap maps were computed as a function of the diameter $d_{3}$ of the central inclusion. The obtained results are reported in
Fig. 6. At $d_{3} / a=0$, the band gaps correspond to those obtained for the simple boron nitride lattice with the same hole diameters. This operating point is marked by a solid line in Figs. 5(c) and 5(d) and corresponds to $d_{2} / a=0.7$. For $d_{3} / a$ $=0.3$, a phononic fractional bandwidth of $39.2 \%$ is obtained, while the photonic fractional bandwidth is at the same time $12.7 \%$ for TE polarization and $4.5 \%$ for TM polarization. Though of smaller extent, the TM band gap is located at higher optical frequencies, so that larger holes can be employed for a fixed optical wavelength. With $\lambda=1550 \mathrm{~nm}$, the pitch is $a=981 \mathrm{~nm}$, and the holes diameters are $d_{1}$ $=1177 \mathrm{~nm}, d_{2}=687 \mathrm{~nm}$, and $d_{3}=294 \mathrm{~nm}$. In turn, the phononic band gap is found as low as $f=1.1 \mathrm{GHz}$ at its center.

Tables I and II summarize, when applicable, reasonable geometrical parameters resulting in the opening of phoxonic band gaps for TE and TM polarized waves, respectively. Priority has been given to structures exhibiting photonic band gaps at high optical frequencies and phononic band gaps at low acoustic frequencies, as previously mentioned. Numerical examples have been taken for a wavelength $\lambda$ $=1550 \mathrm{~nm}$. None of the presented structures actually exhibits a full photonic band gap, i.e., a simultaneous band gap for both TE and TM electromagnetic waves. Phoxonic band gaps with high fractional bandwidth can however be obtained for each particular polarization, especially with complex hexagonal geometries. In all cases, the elastic frequencies involved remain below $3 \mathrm{GHz}$ and the hole diameters are of the order of a few hundreds of nanometers Interhole spacing can be made larger than $50 \mathrm{~nm}$. These values indicate that such lithium niobate phoxonic crystals are feasible.

\section{CONCLUSION}

The possibility to open phoxonic, i.e., simultaneously phononic and photonic, band gaps in lithium niobate has been theoretically demonstrated. This piezoelectric material is highly acoustically anisotropic, but has low refractive indices as compared with silicon, for instance. Both photonic and phononic gap maps of $2 \mathrm{D}$ crystals made of cylindrical air holes were computed and compared for different lattice

TABLE I. Summary of the best phoxonic crystal configurations identified for the various lattice and unit cell geometries considered in this paper. Photonic band gaps are given for the TE polarization only, while phononic band gaps are valid for all polarization components. $\lambda$ is the optical wavelength. $a$ is the pitch of the structure. $d_{1}, d_{2}$, and $d_{3}$ are the diameters of the various inclusions. Center frequencies of phononic band gaps are given for $\lambda=1550 \mathrm{~nm}$.

\begin{tabular}{|c|c|c|c|c|c|c|c|}
\hline & Lattice & $a / \lambda$ & $d_{1} / \lambda$ & $d_{2} / \lambda$ & $d_{3} / \lambda$ & $\begin{array}{l}\text { Relative photonic band gap } \\
(\%)\end{array}$ & $\begin{array}{l}\text { Relative phononic } \\
\text { band gap @ } \\
\text { center frequency }\end{array}$ \\
\hline 1 & Square & 0.395 & 0.380 & $\cdots$ & $\cdots$ & 6.9 & $53.5 \% @ 3.4 \mathrm{GHz}$ \\
\hline 2 & Hexagonal & \multicolumn{6}{|c|}{ No simultaneous photonic and phononic band gap. } \\
\hline 3 & Honeycomb & 0.371 & 0.356 & $\cdots$ & $\cdots$ & 10.1 & 69.9\%@2.1 GHz \\
\hline 4 & Boron nitride & 0.527 & 0.422 & 0.59 & $\cdots$ & 5.1 & 64.3\%@1.4 GHz \\
\hline 5 & Boron nitride & 0.354 & 0.354 & 0.276 & $\cdots$ & 7.4 & $36.8 \% @ 2.2 \mathrm{GHz}$ \\
\hline 6 & Boron nitride & 0.531 & 0.637 & 0.372 & $\cdots$ & 2.4 & 51\%@1.4 GHz \\
\hline 7 & Boron nitride & 0.62 & 0.868 & 0.335 & $\cdots$ & 4.6 & 18.4\%@1.0 GHz \\
\hline 8 & Heterometric hexagonal & 0.678 & 0.542 & 0.678 & 0.271 & 2.2 & $20.8 \% @ 1.1 \mathrm{GHz}$ \\
\hline 9 & Heterometric hexagonal & 0.354 & 0.354 & 0.255 & 0.071 & 7.4 & 19.4\%@2.2 GHz \\
\hline 10 & Heterometric hexagonal & 0.388 & 0.466 & 0.272 & 0.116 & 12.7 & $39.2 \% @ 1.8 \mathrm{GHz}$ \\
\hline 11 & Heterometric hexagonal & 0.616 & 0.862 & 0.246 & 0.123 & 5.5 & $6.3 \% @ 2.0 \mathrm{GHz}$ \\
\hline
\end{tabular}


TABLE II. Summary of the best phoxonic crystal configurations identified for the various lattice and unit cell geometries considered in this paper. Photonic band gaps are given for the TM polarization only, while phononic band gaps are valid for all polarization components. $\lambda$ is the optical wavelength. $a$ is the pitch of the structure. $d_{1}, d_{2}$, and $d_{3}$ are the diameters of the various inclusions. Center frequencies of phononic band gaps are given for $\lambda=1550 \mathrm{~nm}$.

\begin{tabular}{|c|c|c|c|c|c|c|c|}
\hline & Lattice & $a / \lambda$ & $d_{1} / \lambda$ & $d_{2} / \lambda$ & $d_{3} / \lambda$ & $\begin{array}{l}\text { Relative photonic } \\
\text { band gap } \\
(\%)\end{array}$ & $\begin{array}{c}\text { Relative phononic } \\
\text { band gap @ } \\
\text { center frequency }\end{array}$ \\
\hline 1 & Square & \multicolumn{6}{|c|}{ No simultaneous photonic and phononic band gap. } \\
\hline 2 & Hexagonal & 0.497 & 0.457 & $\cdots$ & $\cdots$ & 15.1 & $15.5 \% @ 2.5 \mathrm{GHz}$ \\
\hline 3 & Honeycomb & \multicolumn{6}{|c|}{ No simultaneous photonic and phononic band gap. } \\
\hline 4 & Boron nitride & 0.6 & 0.48 & 0.624 & $\cdots$ & 3 & 46.8\%@1.3 GHz \\
\hline 5 & Boron nitride & 0.825 & 0.825 & 0.759 & $\cdots$ & 2.3 & 70.1\%@0.9 GHz \\
\hline 6 & Boron nitride & 0.625 & 0.75 & 0.438 & $\cdots$ & 4.5 & $51 \% @ 1.2 \mathrm{GHz}$ \\
\hline 7 & Boron nitride & 0.272 & 0.381 & 0.147 & $\cdots$ & 12.8 & 18.4\%@2.2 GHz \\
\hline 8 & Heterometric hexagonal & 0.628 & 0.502 & 0.628 & 0.251 & 1.8 & 20.8\%@1.2 GHz \\
\hline 9 & Heterometric hexagonal & 0.589 & 0.589 & 0.424 & 0.118 & 2.7 & 19.4\%@1.3 GHz \\
\hline 10 & Heterometric hexagonal & 0.633 & 0.76 & 0.443 & 0.19 & 4.5 & 39.2\%@1.1 GHz \\
\hline 11 & Heterometric hexagonal & 0.264 & 0.37 & 0.106 & 0.053 & 16.6 & 6.3\%@4.6 GHz \\
\hline
\end{tabular}

geometries. In particular, single-atom hexagonal and square lattices, as well as multiple-atom lattices based on the honeycomb geometry were considered. We have observed that the larger photonic and phononic band gaps are generally not obtained for the same choice of structure. The best compromise has been reached by considering a hexagonal symmetry with three different atoms in the unit cell, i.e., by decreasing the symmetry of the periodic structure. The low refractive index of the material however prevents from opening complete phoxonic band gaps, as the gap maps for the optical TE and TM polarizations do not overlap in any of the considered structures. The simultaneous confinement of both elastic and electromagnetic energy in a same volume and at common wavelength scale remains however possible, provided that the incident light is polarized. These results are independent of the scale of the structure, provided optical and acoustic wavelengths are scaled proportionally with the pitch of the array. For an optical wavelength of $1550 \mathrm{~nm}$, holes in the hexagonal lattice with three atoms are of sub micrometer diameter. The smallest separation between them can be kept larger than $50 \mathrm{~nm}$, well within the range of nanoscale fabrication techniques, and the acoustic frequencies are in the gigahertz range. Phoxonic crystals hold promises for the simultaneous confinement and tailoring of sound and light waves in very tiny volumes, with potential applications to acousto-optical devices and highly controllable photonphonon interactions.

\section{ACKNOWLEDGMENTS}

The authors gratefully acknowledge Abdelkrim Khelif for enlightening discussions. S.S.S. acknowledges support from the Région de Franche-Comté.

${ }^{1}$ E. Yablonovitch, Phys. Rev. Lett. 58, 2059 (1987).

${ }^{2}$ E. Yablonovitch, J. Opt. Soc. Am. B 10, 283 (1993).

${ }^{3}$ M. S. Kushwaha, P. Halevi, L. Dobrzynski, and B. Djafari-Rouhani, Phys. Rev. Lett. 71, 2022 (1993)

${ }^{4}$ T. Gorishnyy, C. K. Ullal, M. Maldovan, G. Fytas, and E. L. Thomas,
Phys. Rev. Lett. 94, 115501 (2005).

${ }^{5}$ V. Laude, A. Khelif, S. Benchabane, M. Wilm, T. Sylvestre, B. Kibler, A. Mussot, J. M. Dudley, and H. Maillotte, Phys. Rev. B 71, 045107 (2005).

${ }^{6}$ M. M. de Lima, Jr., R. Hey, and P. V. Santos, Appl. Phys. Lett. 83, 2997 (2003).

${ }^{7}$ M. Trigo, A. Bruchhausen, A. Fainstein, B. Jusserand, and V. ThierryMieg, Phys. Rev. Lett. 89, 227402 (2002).

${ }^{8}$ P. Dainese, P. Russell, N. Joly, J. Knight, G. Wiederhecker, H. L. Fragnito, V. Laude, and A. Khelif, Nat. Phys. 2, 388 (2006).

${ }^{9}$ P. Dainese, P. Russell, G. Wiederhecker, N. Joly, H. Fragnito, V. Laude, and A. Khelif, Opt. Express 14, 4141 (2006).

${ }^{10}$ J.-C. Beugnot, T. Sylvestre, H. Maillotte, G. Melin, and V. Laude, Opt. Lett. 32, 17 (2007).

${ }^{11}$ M. Maldovan and E. Thomas, Appl. Phys. B: Lasers Opt. 83, 595 (2006).

${ }^{12}$ M. Maldovan and E. Thomas, Appl. Phys. Lett. 88, 251907 (2006).

${ }^{13}$ A. V. Akimov, Y. Tanaka, A. B. Pevtsov, S. F. Kaplan, V. G. Golubev, S. Tamura, D. R. Yakovlev, and M. Bayer, Phys. Rev. Lett. 101, 033902 (2008).

${ }^{14}$ M. Roussey, M. Bernal, N. Courjal, and F. Baida, Appl. Phys. Lett. 87, 241101 (2005).

${ }^{15}$ S. Benchabane, A. Khelif, J.-Y. Rauch, L. Robert, and V. Laude, Phys. Rev. E 73, 065601(R) (2006).

${ }^{16}$ N. G. R. Broderick, G. W. Ross, H. L. Offerhaus, D. J. Richardson, and D. C. Hanna, Phys. Rev. Lett. 84, 4345 (2000).

${ }^{17}$ G. Kovacs, M. Anhorn, H. E. Engan, G. Visintini, and C. C. W. Ruppel, Proc.-IEEE Ultrason. Symp. 1, 435 (1990).

${ }^{18}$ D. F. Nelson and R. M. Mikulyak, J. Appl. Phys. 45, 3688 (1974).

${ }^{19}$ M. Wilm, S. Ballandras, V. Laude, and T. Pastureaud, J. Acoust. Soc. Am. 112, 943 (2002).

${ }^{20}$ T.-T. Wu, Z.-G. Huang, and S. Lin, Phys. Rev. B 69, 094301 (2004).

${ }^{21}$ V. Laude, M. Wilm, S. Benchabane, and A. Khelif, Phys. Rev. E 71, 036607 (2005)

${ }^{22}$ A. Khelif, B. Aoubiza, S. Mohammadi, A. Adibi, and V. Laude, Phys. Rev. E 74, 046610 (2006).

${ }^{23}$ Z. Zhang and S. Satpathy, Phys. Rev. Lett. 65, 2650 (1990).

${ }^{24}$ K. M. Leung and Y. F. Liu, Phys. Rev. Lett. 65, 2646 (1990).

${ }^{25}$ Available at www.rsoftdesign.com.

${ }^{26}$ F. Baida, D. Van Labeke, G. Granet, A. Moreau, and A. Belkhir, Appl. Phys. B: Lasers Opt. 79, 1 (2004).

${ }^{27}$ D. Caballero, J. Sanchez-Dehesa, C. Rubio, R. Martinez-Sala, J. V. Sanchez-Perez, F. Meseguer, and J. Llinares, Phys. Rev. E 60, R6316 (1999).

${ }^{28}$ C. M. Anderson and K. P. Giapis, Phys. Rev. Lett. 77, 2949 (1996).

${ }^{29}$ D. Cassagne, C. Jouanin, and D. Bertho, Phys. Rev. B 53, 7134 (1996).

${ }^{30}$ K. M. Ho, C. T. Chan, and C. M. Soukoulis, Phys. Rev. Lett. 65, 3152 (1990).

${ }^{31}$ P. R. Villeneuve and M. Piche, Phys. Rev. B 46, 4969 (1992). 